\title{
Comparison of two rodent models of maternal separation on juvenile social behavior
}

\section{Betty Zimmerberg* and Kristin A. Sageser}

Department of Psychology, Williams College, Williamstown, MA, USA

\section{Edited by:}

Josephine Johns, University of North

Carolina at Chapel Hill, USA

\section{Reviewed by:}

Elizabeth McCone Byrnes, Tufts University Cummings School of Veterinary Medicine, USA

Kelly Lambert, Randolph-Macon

College, USA

\section{*Correspondence:}

Betty Zimmerberg, Department of

Psychology, Williams College,

18 Hoxsey Street, Williamstown, MA 01267, USA.

e-mail: betty.zimmerberg@

williams.edu
Early childhood deprivation is associated with an increased risk of attachment disorders and psychopathology. The neural consequences of exposure to stress early in life have used two major rodent models to provide important tools for translational research. Although both models have been termed maternal separation (MS), the paradigms differ in ways that clearly shift the focus of stress between maternal and offspring units. The first model, here called early deprivation (ED), isolates pups individually while the dam is left not alone, but with a subset of littermates in the home nest ("stay-at-homes"). The other model, here called MS, isolates the dam in a novel cage while the pups are separated together. In this study, these two early stress models were directly compared for their effects on social behaviors in male and female juvenile offspring. Although both models altered play behavior compared to controls, patterns of prosocial behaviors versus submissive behaviors differed by model and sex. Additionally, there were main effects of sex, with female ED subjects exhibited masculinizing effects of early stress during play sessions. Maternal behavior upon reunion with the isolated subjects was significantly increased in the MS condition compared to both ED and control conditions, which also differed but by a lesser magnitude. "stay-at-homes" were tested since some laboratories use them for controls rather than undisturbed litters; they displayed significantly different sex-dependent play compared to undisturbed subjects. These results indicate that early stress effects vary by paradigm of separation. We suggest that MS produces greater stress on the dam and thus greater maternal mediation, while ED causes greater stress on the neonates, resulting in different behavioral sequela that warrant attention when using these models for translational research.

Keywords: maternal separation, early deprivation, neonatal isolation, play behavior, maternal behavior, social behavior, isolation, stress

\section{INTRODUCTION}

Early traumatic experiences produce long-term neural changes that are implicated in the etiology of psychiatric disorders (Hofer, 1996; Sanchez et al., 2001; Teicher et al., 2002). Children who have been the victims of physical or sexual abuse are at significantly greater risk for mental illness in adulthood (Bifulco et al., 1991; Brown and Anderson, 1991; Caspi et al., 2003). Social behavior subsequent to early stress has been studied less frequently than psychopathology. Children with histories of severe neglect have been shown to have attachment disorders and altered social behavior (O'Connor and Rutter, 2000; Zeanah, 2000; Zeanah et al., 2002). For example, children who had spent at least 8 months in a Romanian orphanage displayed significantly increased levels of indiscriminate friendliness compared to non-adopted children or children who had been institutionalized 4 months or less (Chisholm, 1998).

Animal models of child neglect have been developed to mimic the experience of isolation stress in children and can be used to study the molecular mechanisms of its long-term consequences on social behavior. In rats, the neonatal pup is dependent upon the mother for thermoregulation, nutrition, stimulation of urination and protection for the first 2 weeks of life (Sanchez et al.,
2001). Early separation from the dam has been demonstrated to affect a myriad of physiological systems in the neonate, including alterations in heart rate, circadian rhythms, and levels of circulating hormones (Hofer, 1987; Stanton et al., 1988; Kuhn et al., 1990; Stanton and Levine, 1990). Separation of pups is also associated with amplified neural CRF gene expression, elevated corticosterone, and neurosteroid levels (Francis et al., 1999; Kehoe et al., 2000; Frisone et al., 2002). Later in life, these animals show greater activation of the hypothalamic-pituitary-adrenal (HPA) axis (Francis et al., 1999). Behavioral sequela from neonatal isolation stress have also been well documented. For example, adult rats that experienced separation as pups exhibit more anxiety, less aggression, and less maternal behavior (Boccia and Pedersen, 2001), groom more (Zimmerberg et al., 1999), and display learning deficits (Lehmann et al., 1999; Frisone et al., 2002; Sandstrom and Hart, 2005).

Maternal behavior upon reunion with separated pups has been proposed to play a significant role in the moderation of neuroendocrine responses to stress, cognitive development, and social learning. Offspring that received increased licking and grooming (LG) from the dam during the first 10 days of life showed enhanced 
hippocampal development and spatial learning and memory (Liu et al., 2000), and decreased HPA responses to stress (Liu et al., 1997). Juvenile male rats receiving decreased dam LG displayed more play fighting compared to increased LG offspring (Parent and Meaney, 2008). Similarly, as adults, low LG males were more aggressive and defensive during a resident intruder test (Menard and Hakvoort, 2007). Pups reared with complete maternal deprivation showed impaired performance in social learning tasks (Levy et al., 2003). In this study, pups were removed from the nest at PN4 and fed via a gastronomy tube. Maternal deprivation did not affect adult performance on non-social learning tasks (water maze and radial arm maze); however, performance was impaired on all three social learning tasks. Animals reared artificially made no distinction between a familiar and non-familiar conspecific, and did not develop a preference for food previously eaten by a familiar conspecific, while animals reared with their mothers did. Artificially reared females also responded less rapidly to pups than did females reared with their mothers. These results imply that animals reared in the absence of maternal care, even when provided with simulated forms of care, develop significant impairments in social behavior as adults.

While an expanding body of literature examines the effects of early isolation-induced stress in the rat, there is little uniformity in the paradigms used to define this type of early stress between laboratories or in the reported outcomes (Lehmann and Feldon, 2000). Manipulation procedures range from brief daily separation of pups ("early handling"), to periods of single or repeated separations for 1-24 h [alternately called "early deprivation (ED)," "neonatal social isolation" or "maternal separation (MS)"], to complete separation with artificial rearing from birth to weaning. Pups might be isolated or separated as a total litter. The age of the pups and the number of days during which these procedures are carried out also varies between laboratories. The body temperature of separated pups in different paradigms may also vary and affect outcome (Zimmerberg and Shartrand, 1992).

Thus the purpose of this study was not only to investigate the effects of early social isolation-induced stress on subsequent social behavior during adolescence in rats, but also to determine whether the two major models of neglect would differ in outcome. We used the isolation procedure of our own laboratory ("ED," e.g., Pryce et al., 2003; Zimmerberg et al., 2003) and the most typical alternative procedure, "MS" (e.g., Francis et al., 1999). In the ED model, pups are isolated individually while the dam is left with some littermates in the home nest. In the (MS paradigm, the dam is isolated in a novel environment while the pups remain together in the home nest. In these two situations both the pups and the dams are exposed to very different experiences during the separation period. Not only are the pups subjected to two distinct environments in ED versus MS, but the dams also have two distinct environments.

Our behavioral measure, play fighting, also called rough-andtumble play, is the most common form of social behavior in juvenile rodents, and involves non-antagonistic chasing, wrestling, nipping, and hitting. While the behaviors of play fighting mimic adult aggression, the participants in play fighting are almost never seriously injured. Play is solicited by the area of attack; in play, attacks involve an attempt to rub the snout into the nape of the partner, while serious fighting involves biting and is targeted at the lower flanks and dorsum or the face (Pellis and Pellis, 1998). Attempted nape contacts are usually resisted by the recipient, who will adopt defensive tactics to avoid the contact or counterattack with its own attempt to contact the partner's nape. A common defensive tactic is the supine position. In response to an attack, the defender rotates into the supine position and uses its paws to hold off the attacker. When the attacking rat attempts to contact the nape while the other is in the supine position, it is called a pin. Other defensive tactics include evading the contact of the attacker by moving away, or lifting the front paws off the ground while facing the attacker to meet the attack head-on, called boxing. In rough-and-tumble play there are often many role reversals as partners alternate between attacking and defending.

Frequency of play bouts in rats is age-dependent, beginning at about 18 days and reaching peak levels in the fifth week of life. Play then declines as the animal approaches sexual maturity (Pellis and Pellis, 1990). There are also sex differences in play, both in the frequency and composition of play behavior. Males play more than females, and not only initiate more playful contacts, but are also more likely than females to respond to such playful contacts. When females do respond, they are more likely to evade, while males are more likely to turn supine. As play fighting consists of an attack and a defense, male pairs play fight more than female pairs, and mixed-pairs display an intermediate level of play (Pellis and Pellis, 1990). These differences have been attributed to an interaction of sensory, motor, and motivational differences between sexes (Pellis et al., 1997).

The two previous studies in rats examining the effects of early separation stress on play behaviors have both used the MS paradigm, with contradictory results. A 3-h daily MS paradigm during the first 2 weeks of life found overall levels of playfulness were not affected, but that responsiveness to playful contacts (with evasions or partial rotations) was enhanced in males in a pattern that suggested feminization (Arnold and Siviy, 2002). In a more recent study that also examined the effect of the MS paradigm on juvenile play, but only in males, previously separated subjects displayed more aggressive play (attacks, pulling, and biting) and less submissive play (supine, evading) than controls (Veenema and Neumann, 2008).

In this experiment, the ED and MS models were directly compared for their effects on play behavior in both male and female juvenile subjects. Although each model consisted of daily separations of equal length for 2 weeks, starting and ending on the same postnatal days for all subjects, we hypothesized that these two manipulations might not result in identical behavioral sequela. Since MS effects might be mediated by maternal stress, while ED effects due to more direct effects of isolation, we hypothesized a greater level of altered play level in ED compared to MS and control subjects. If MS subjects did differ from controls, play disruption might be seen in submissive measures since previous studies report enhanced stress reactivity in HPA function with the MS paradigm. In addition, we looked for behavioral differences in within-litter non-isolated subjects since they are sometimes used as control subjects in other laboratories rather than distinct undisturbed control litters. 


\section{MATERIALS AND METHODS SUBJECTS}

Subjects were bred in the Williams College animal facility from female and male Long-Evans rats (Harlan Sprague-Dawley, Indianapolis, IN, USA). All procedures were approved by the Williams College Institutional Animal Care and Use Committee. After detection of a vaginal plug, females were individually housed in plastic cages in a separate nursery room maintained on a 12:12 light:dark schedule, at $22^{\circ} \mathrm{C}, 55 \%$ humidity. Females were inspected three times daily for births; the day that birth was observed was designated as postnatal day (PN) 0 . On the day following their birth (PN1), litters were sexed, and culled to 12 if necessary, with 6 males and 6 females when possible. On PN2, litters were randomly assigned to one of three conditions: ED, MS, or Control. All litters experienced the same standard nursery care, consisting of twice weekly bedding changes starting on PN3.

All subjects were ear-punched for identification on PN12, and weaned and weighed at PN25, when the subjects were removed from the nursery and rehoused in hanging cages in same-sex pairs. On PN29, subjects were rehoused individually prior to testing to produce robust play behavior (Thor and Holloway, 1984).

A total of 106 subjects were tested in Experiment 1, as follows: ED: 16 males and 16 females, representing 8 distinct litters; MS: 18 males and 22 females, representing 11 distinct litters, and Controls: 18 males and 16 females, representing 9 distinct litters. In addition to the above subjects, 16 males and 16 females from the 8 ED litters who had remained with the dams while the ED subjects were separated were also observed for play behavior (within-litter non-isolated subjects, or "stay-at-homes").

\section{APPARATUS}

Behavioral testing was conducted in a cylindrical glass chamber $(30 \mathrm{~cm}$ diameter $\times 30 \mathrm{~cm}$ high $)$ housed within a soundattenuating box. A video camera was attached to the ceiling of the box. The only lighting in the room was from a $60-\mathrm{W}$ red light bulb and the computer monitor.

\section{NEONATAL PROCEDURES \\ Early deprivation}

Daily from PN2 through PN14, at 1300 h, eight pups (four male and four female) were removed from the home cage, leaving the four "stay-at-home" pups with the dam during the separation period. The home cage with the dam and "stay-at-home" pups were placed back in the same position on a shelf in the nursery. Subjects were placed individually in plastic cups $(10.6 \mathrm{~cm}$ diameter) and transported to an adjacent room. Cups were placed in a heated, circulating water bath set at $34^{\circ} \mathrm{C}$ to mimic vestibular and thermal stimulation experienced in the nest. Rectal temperature readings in prior experiments had assured that body temperatures were normative at $34^{\circ} \mathrm{C}$ (Zimmerberg et al., 2003). After $3 \mathrm{~h}$, subjects were returned to the home cage. Subjects were weighed on the first and last days of isolation.

\section{Maternal separation}

Daily from PN2 through PN14, at $1300 \mathrm{~h}$, the dam was removed from the home nest and placed in a novel cage with fresh bedding. The cage was placed in a new position on the top shelf in the nursery room. The entire litter was transported in the home cage, which was placed on a heating pad set at $32^{\circ} \mathrm{C}$ in an adjacent room. Rectal temperature readings in pilot studies had assured that body temperatures were normative $\left(34^{\circ} \mathrm{C}\right)$. After $3 \mathrm{~h}$ of separation the home cage was returned to the nursery and the dam returned to the nest. Subjects were weighed on the first and last days of separation.

\section{Controls}

Control litters were left undisturbed with the exception of weighing and earpunching on days equivalent to the designated weighing and earpunching days for the ED and MS groups.

\section{BEHAVIORAL PROCEDURES \\ Maternal behavior observations}

Dams were observed in the nursery $10 \mathrm{~min}$ after the pups were returned to their home nest on the seventh day of the neonatal separation procedures for $1 \mathrm{~min}$. For the Control condition, observations were made at equivalent times. The observer noted whether the dam has relocated the "nest," whether the dam was still licking pups, and whether the dam was passively nursing (supine).

\section{Play behavior testing}

Subjects were habituated to the play-testing chamber on PN31 by placing them individually into the chamber for $30 \mathrm{~min}$. On PN32, two subjects (same-sex, same-litter cage-mates) were placed inside the testing chamber. Subjects were placed in the play chamber for 6 min on PN32; no recordings were made on this first day. After the play session, subjects were returned to their individual cages. Play sessions continued in the same manner on PN33 and 34, 6 min each day, but on these days behaviors were recorded. All testing took place between 1000 and $1400 \mathrm{~h}$.

Video recordings of each play session were scored for number of occurrences and duration of eight different behaviors for each subject using computerized observation software (LabTimer). Behaviors scored were attacks, pins, supine postures, evasions, boxing bouts, sniffing, submissions, and rearing. An attack was defined as a contact of the snout to the nape or dorsum of the partner. Supine occurrences were defined as when a subject rotated into the supine position and used its paws to defend itself, while pinning was defined as when a subject attempted to contact the nape while his partner was in the supine position. Evasion was defined as moving away from an attack without assuming the supine position, and boxing was defined as lifting the front paws to counter the partner's attack. Sniffing included sniffing of any region of the partner. Submission was defined as when an attacked subject made no attempt to evade or assume the supine position, and remained immobile during the attack.

\section{Data analysis}

Play data were analyzed by analysis of variance (ANOVA) with postnatal treatment condition (ED, MS, and Control) and sex as the independent variables, and day of testing as a repeated measure. Within-litter "stay-at-home" subjects were compared to subjects from Control litters in a separate ANOVA. Significant main effects were further analyzed with Fisher's LSD test, and significant interactions further analyzed with Means Comparison Tests ( $p$ 's $<0.05$ criteria). Categorical maternal behavior data 
were analyzed by the independent-samples Kruskal-Wallis tests ( $p$ 's $<0.05$ criteria).

\section{RESULTS \\ BODY WEIGHT}

Neonatal separation, whether ED or MS, had no significant effect on body weight or rate of growth from PN2-14. Weight gain for the ED, MS, and Control conditions were $21.9 \pm 1.8,23.7 \pm 0.8$ and $21.5 \pm 0.5 \mathrm{~g}$, respectively.

\section{MATERNAL BEHAVIOR}

There was a significant main effect of postnatal treatment condition on overall maternal behavior ( $p$ 's $<0.05$ ). The MS condition dams displayed significantly more maternal behavior overall than the ED condition dams, which in turn displayed significantly more overall maternal behavior than Control condition dams. All MS dams relocated their "nests" while only 3 ED dams did so; Control dams never moved their nest. Postnatal treatment also had a significant effect on the likelihood of dams to still be licking pups at 10 min post-reunion, with $8 / 11 \mathrm{MS}$ dams were still engaged in licking compared to 3/8 ED dams and 0/9 Control dams. There was a significant effect of postnatal treatment on the likelihood of dams to take longer than $10 \mathrm{~min}$ post-reunion to lie supine (passive nursing). Only $3 / 11$ of the MS dams were supine compared to $5 / 8$ of the ED dams and all nine of the Control dams $10 \mathrm{~min}$ post-reunion.

\section{PLAY BEHAVIOR: ED, MS, AND CONTROL GROUPS}

The effects of postnatal treatment and sex on play behavior measures observed over 2 days of testing are seen in Table 1. The results are also presented in Figure $\mathbf{1}$ as the percent change from control so that the overall pattern of results can be seen.

There was a significant main effect of postnatal treatment on the number of attacks, $F(2,200)=3.341, p=0.04$. ED subjects

Table 1 | The effects of early deprivation and maternal separation on mean number (+SEM) and mean total duration (seconds + SEM) of play behavior measures in adolescent rats.

\begin{tabular}{lccc}
\hline Behavior & $\begin{array}{l}\text { Early } \\
\text { deprivation }\end{array}$ & $\begin{array}{l}\text { Maternal } \\
\text { separation }\end{array}$ & Control \\
\hline Attacks & $27.14+1.95^{*}$ & $24.51+1.65$ & $21.21+1.56$ \\
Attack duration & $45.47+4.17^{*}$ & $35.94+2.80$ & $31.85+3.10$ \\
Boxing bouts & $2.89+0.38^{* *}$ & $1.43+0.20^{*}$ & $2.28+0.28$ \\
Boxing duration & $3.51+0.46^{*}, *$ & $1.47+0.25^{*}, * *$ & $2.43+0.40$ \\
Pins & $8.58+0.85$ & $9.59+0.85$ & $8.41+0.88$ \\
Pinning duration & $28.93+3.08$ & $31.53+3.01$ & $28.19+3.38$ \\
Evasions & $18.72+1.71^{*}$ & $16.71+1.38^{*}$ & $11.56+1.09$ \\
Evasion duration & $23.58+2.38^{*}, * *$ & $18.35+1.71 * * *$ & $13.02+1.48$ \\
Supine postures & $9.09+0.94$ & $10.27+0.91$ & $8.74+0.93$ \\
Supine duration & $29.58+3.21$ & $34.02+3.21$ & $29.79+3.56$ \\
Submissions & $4.84+0.91$ & $3.90+0.77$ & $5.5+1.00$ \\
Submissive duration & $21.04+1.67$ & $19.86+1.51$ & $17.43+1.59$ \\
\hline
\end{tabular}

* Significantly different from control.

**ED and MS significantly different. attacked significantly more than Control subjects, while MS subjects did not differ from either ED or Control subjects. Sex also had a significant main effect on the number of attacks, $F$ (1, $200)=3.996, p=0.05$, with males $(25.80+1.45)$ attacking more than females $(22.50+1.35)$. Of subjects that performed at least one attack [all except four subjects (1 ED, 2 MS, 1 Control)], postnatal treatment had a significant main effect on the total duration of attacks per play session, $F(2,196)=5.276, p=0.006$. ED subjects attacked significantly longer than MS and Control subjects, which did not differ from each other. Postnatal treatment also had a significant main effect on the duration of a single attack, $F(2,196)=4.573, p=0.01$. In this case, ED subjects attacked significantly longer per attack $(1.54+0.11)$ than Control subjects $(1.33+0.05)$. There were no differences between ED and MS $(1.42+0.07)$ or MS and Control subjects in the duration per attack.

There was a significant main effect of postnatal treatment condition on the number of boxing bouts, $F(2,200)=6.691$, $p=0.002$. ED and Control subjects both boxed significantly more often than MS subjects, and there was no significant difference between ED and Control subjects. There was also a significant interaction of postnatal treatment and sex, $F(2,200)=3.016$, $p=0.05$ (see Figure 2A). ED females and Control females both boxed significantly more than MS females. There was no difference between ED and Control females or between ED, MS, and Control males. MS females also boxed significantly less than MS males.

A similar pattern was seen in the total duration of boxing per play session. There was a significant main effect of postnatal treatment condition on the total boxing duration, $F(2,200)=7.83$, $p=0.0005$. ED subjects boxed longest, with significantly greater duration than both MS and Control subjects. Control subjects boxed significantly longer than MS subjects, which had the shortest duration. An interaction of postnatal treatment and sex also significantly affected the boxing duration, $F(2,200)=4.917, p=0.008$, and revealed that the main effect seen was due to differences in females only (see Figure 2B). ED females boxed significantly longer than both Control and MS females, and Control females boxed significantly longer than MS females. ED females also boxed significantly longer than ED males, while MS females boxed for a significantly shorter duration than MS males. Control males and females did not differ.

Of the 148 subjects who engaged in at least one boxing bout, there was a significant main effect of postnatal treatment on the mean duration of a boxing bout, $F(2,136)=3.779, p=0.03$. ED subjects boxed significantly longer $(1.35+0.20 \mathrm{~s}$ per bout $)$ than both Control and MS subjects $(0.94+0.06$ versus $0.97+0.05 \mathrm{~s}$ per box, respectively); there was no significant difference between Control and MS durations. There were twice as many MS subjects who never boxed compared to ED and Controls, which had the same number of non-boxing subjects. An interaction of postnatal treatment and sex also significantly affected the duration per box, $F(2,136)=3.104, p=0.05$. ED females boxed significantly longer per box $(1.61 \pm 0.45 \mathrm{~s})$ than both Control and MS females $(0.83 \pm 0.09$ and $0.83 \pm 0.11 \mathrm{~s}$, respectively). ED females also boxed longer than ED males $(1.07+0.15 \mathrm{~s})$. ED males did no differ from MS or Control males $(1.09 \pm 0.08$ and $0.97 \pm 0.09 \mathrm{~s}$, respectively). 


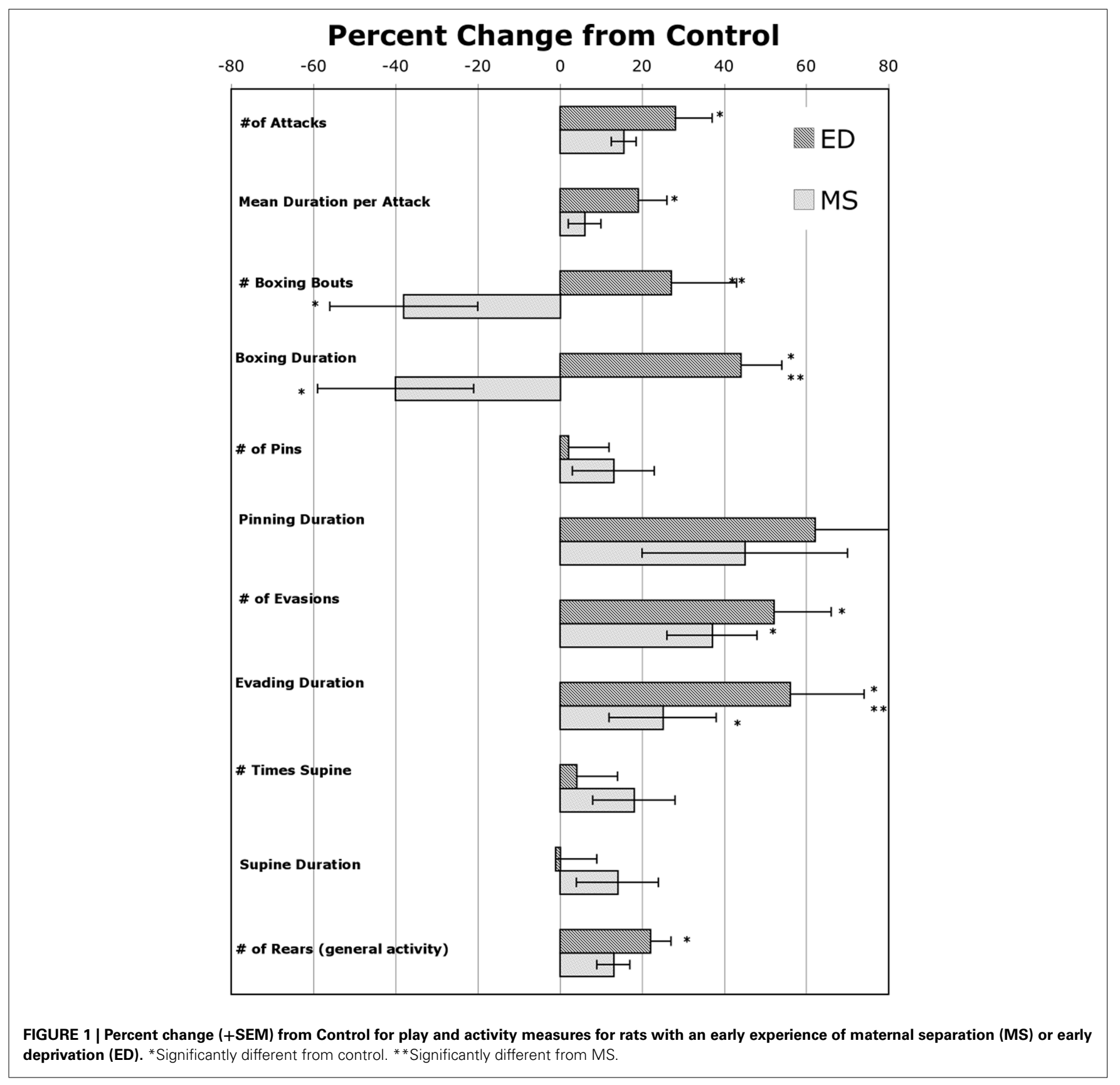

Postnatal treatment condition had a significant main effect on the number of evasions, $F(2,200)=5.853, p=0.003$. ED subjects and MS subjects both evaded significantly more often than Control subjects. There was also a significant main effect of postnatal treatment on the total duration of evasions, $F(2,200)=7.303$, $p=0.0009$. ED subjects evaded significantly longer time than both MS and Control Subjects. MS subjects also evaded significantly longer than Control subjects.

Postnatal treatment condition had a significant main effect on the number of rears, $F(2,200)=3.406, p=0.03$. ED subjects reared significantly more than Control subjects $(31.78+1.34$ compared to $26.07+1.36$ rears per session). There was no significant difference between rears for MS $(29.45+1.27)$ and Control subjects or MS and ED subjects. An interaction of postnatal treatment and sex also significantly affected the number of rears, $F$ (2, $200)=3.165, p=0.04$ (see Figure 3 ). ED males reared significantly more than MS males and also more than Control males. There were no differences in rearing in females. Postnatal treatment condition did not affect rearing duration.

The numbers of pins or pinning duration, supine events or duration, submission events or duration, or sniff events or duration were unaffected by postnatal condition. The day of testing was also not a significant factor in any analyses and data are presented as mean of the 2 days of testing. 

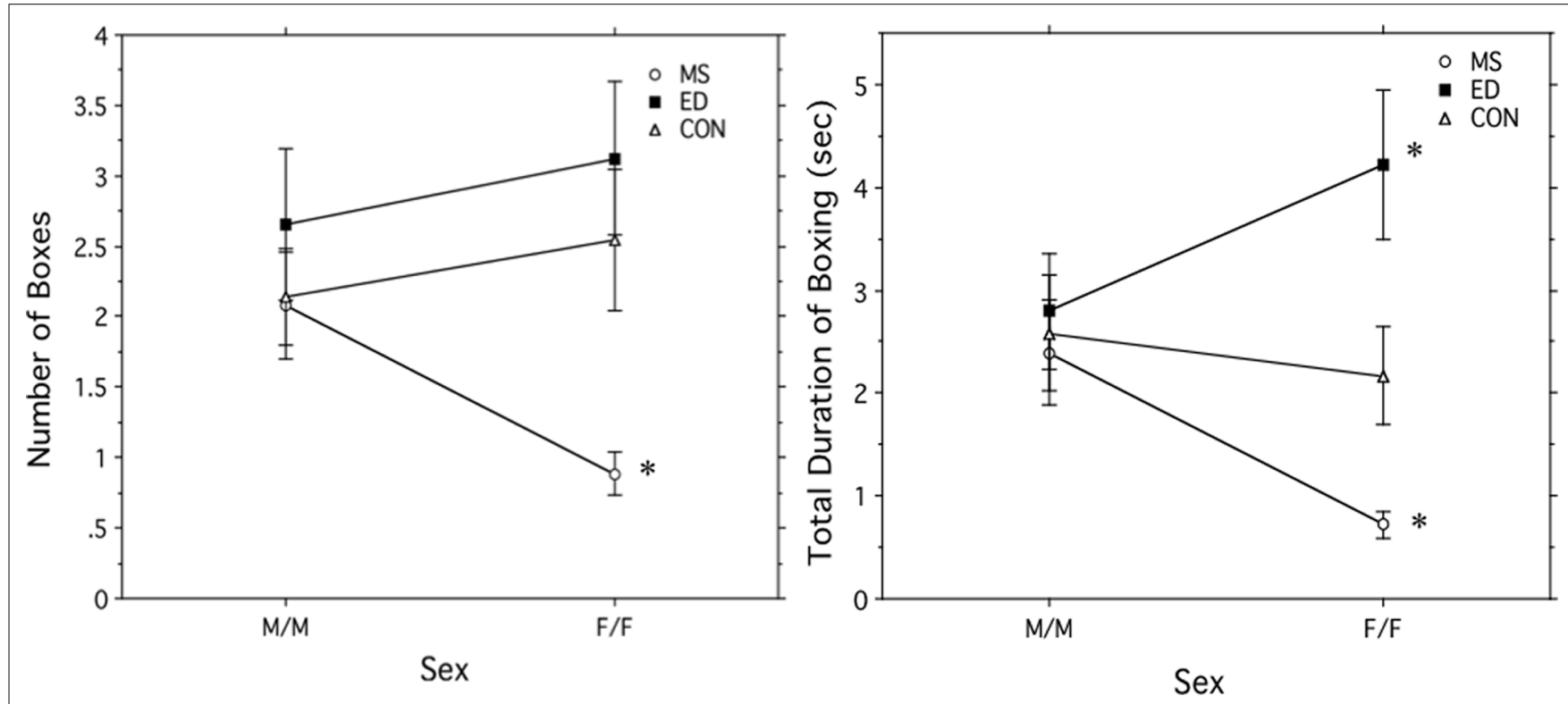

FIGURE 2 | (A) Total number of boxing bouts + SEM and (B) duration of boxing bouts in seconds + SEM in male (M/M) and female (F/F) pairs in one of three postnatal treatment condition: maternal separation (MS), early deprivation (ED), or control. * Significantly different from all other groups.

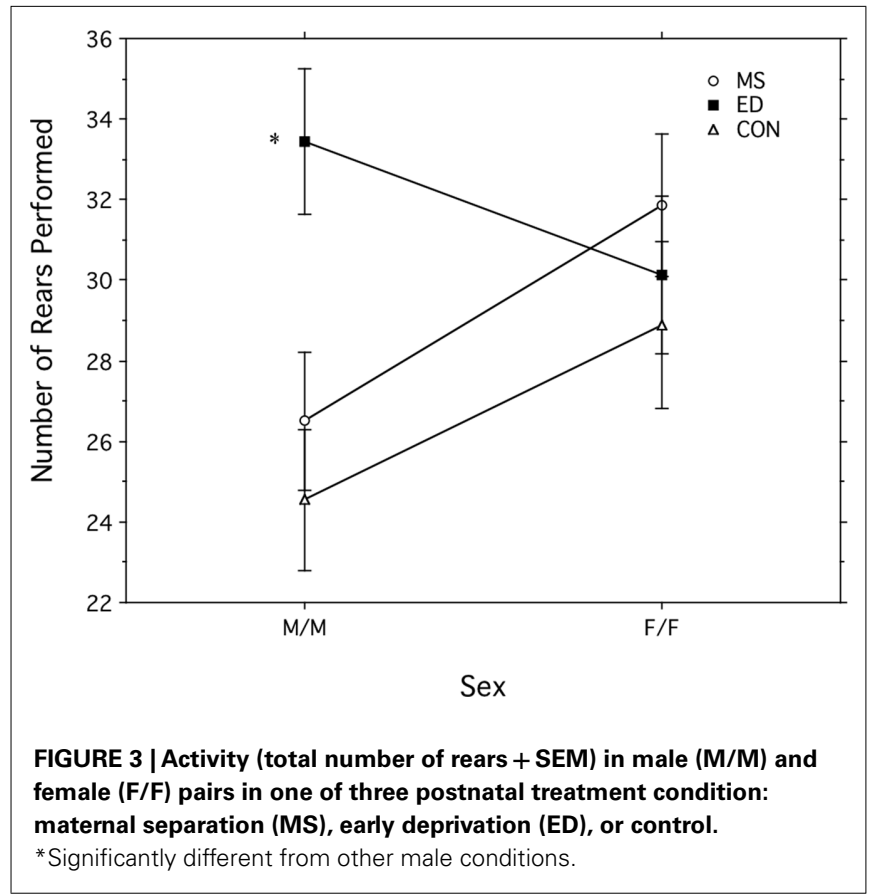

PLAY BEHAVIOR: CONTROL VERSUS STAY-AT-HOME SUBJECTS

There was a consistent pattern of significant interactions between Sex and the type of control group (within-litter Stay-at-home subjects) versus distinct litters (Controls) in play behavior measures, as seen in Table 2. Control males and females did not differ in most measures, Stay-at-home males demonstrated decreased play compared to Control males and Stay-at-home females demonstrated increased play compared to Control females.
Table 2 | Comparison of within-litter ("stay-at-home") and distinct control subjects on mean number (+SEM) and mean total duration (seconds + SEM) of play behavior measures in male and female adolescent rats.

\begin{tabular}{|c|c|c|c|c|}
\hline \multirow[t]{2}{*}{ Behavior } & \multicolumn{2}{|c|}{ Within-litter } & \multicolumn{2}{|c|}{ Distinct litter } \\
\hline & Male & Female & Male & Female \\
\hline Attacks* & $10.1 \pm 1.9$ & $17.8 \pm 4.2$ & $22.6 \pm 2.0$ & $18.6 \pm 2.4$ \\
\hline Attack duration* & $16.9 \pm 2.9$ & $43.9 \pm 7.5$ & $36.7 \pm 4.3$ & $21.7 \pm 3.2$ \\
\hline Boxing bouts & $2.2 \pm 0.5$ & $2.0 \pm 0.5$ & $2.1 \pm 0.3$ & $2.5 \pm 0.5$ \\
\hline Boxing duration & $3.6 \pm 1.3$ & $2.8 \pm 0.7$ & $2.6 \pm 0.5$ & $2.2 \pm 0.5$ \\
\hline Pins* & $2.8 \pm 1.0$ & $10.8 \pm 2.1$ & $8.6 \pm 1.0$ & $8.0 \pm 1.6$ \\
\hline Pinning duration & $13.7 \pm 5.3$ & $29.6 \pm 5.8$ & $28.5 \pm 3.9$ & $27.5 \pm 6.4$ \\
\hline Evasions* & $4.4 \pm 1.2$ & $21.9 \pm 3.3$ & $11.2 \pm 1.3$ & $12.2 \pm 1.9$ \\
\hline Evasion duration* & $6.2 \pm 1.9$ & $25.8 \pm 4.4$ & $13.5 \pm 1.8$ & $12.2 \pm 2.5$ \\
\hline Supine postures* & $3.2 \pm 1.1$ & $12.2 \pm 2.4$ & $8.9 \pm 1.1$ & $8.4 \pm 1.7$ \\
\hline Supine duration & $15.0 \pm 5.7$ & $30.1 \pm 6.0$ & $30.5 \pm 4.3$ & $28.5 \pm 6.5$ \\
\hline Submissions & $3.7 \pm 0.8$ & $3.3 \pm 1.2$ & $6.8 \pm 1.4$ & $3.1 \pm 0.9$ \\
\hline Submissive duration & $5.5 \pm 1.5$ & $5.4 \pm 2.1$ & $18.8 \pm 1.9$ & $14.9 \pm 2.6$ \\
\hline
\end{tabular}

* Significant interaction between condition and sex.

An interaction of postnatal treatment condition and sex significantly affected the number of attacks performed, $F$ (1, $92)=14.743, p=0.0002$. Control males attacked significantly more than Stay-at-home males, while Control females attacked significantly less than Stay-at-home females. Also, Stay-at-home females attacked significantly more than Stay-at-home males, while there was no difference between female and male Controls. There was also a significant main effect of sex on attacks, $F$ (1, $92)=5.798, p=0.02$, with females performing more attacks than 
males. There was also a significant interaction of condition and sex on total attack duration, $F(1,92)=15.635, p=0.0002$, following the same pattern as number of attacks. Of subjects that performed at least one attack, there was a significant main effect of postnatal treatment condition on the mean duration of a single attack, $F(1,86)=14.037, p=0.0003$; Stay-at-home subjects $(1.65+0.07)$ attacked longer per individual attack than Control subjects $(1.35+0.05)$.

An interaction of postnatal treatment condition and sex also significantly affected evasions, $F(1,92)=16.279, p=0.0001$. This interaction followed the same pattern as the interactions seen in attacks: Control males evaded significantly more than Stayat-home males, while Control females evaded significantly less than Stay-at-home females. Also, Stay-at-home females evaded significantly more than Stay-at-home males. There was no difference between Control males and females. An interaction of postnatal treatment condition and sex significantly affected total duration of evasions performed, $F(1,92)=14.232, p=0.0003$. Evasions performed by Control males were significantly longer than evasions performed by Stay-at-home males, while evasions performed by Control females were significantly shorter than evasions performed by Stay-at-home females.

The number of pins was also significantly affected by postnatal treatment condition and sex, $F(1,92)=7.44, p=0.008$. Stay-at-home males pinned significantly less than Control males, and Stay-at-home females pinned significantly more than Stay-athome males, while Control males and females, and Control females and Stay-at-home females did not differ. Total pinning durations did not differ by group condition.

A main effect of sex on mean number of supine occurrences was seen, $F(1,92)=6.414, p=0.01$. Females were supine significantly more times than males during a play session with a mean of $9.93+1.42$ supine events per session, while males were supine only $7.38+0.92$ times on average per session. However, an interaction of postnatal treatment condition and sex revealed that this main effect was due to an effect in Stay-at-home subjects and not Controls, $F(1,92)=7.985, p=0.006$. Following the previously established pattern, Stay-at-home females were supine significantly more than Stay-at-home males. Control males were also supine significantly more than Stay-at-home males, and there was no difference between Control and Stay-at-home females or Control males and Control females.

There was a significant main effect of postnatal treatment condition on the mean number of rears, $F(1,69)=4.103, p=0.05$. Stay-at-home subjects $(31.63+1.91)$ reared more than Control subjects $(26.07+1.37)$. There was no significant effect of postnatal treatment condition on mean total duration of rearing per play session. Group comparisons also did not affect rearing duration, supine duration, submission events or submission duration, or sniff events or sniffing duration.

The only significant effect of day of testing was an interaction with postnatal treatment condition on the mean total duration of boxing per play session, $F(1,92)=5.092, p=0.03$. On PN33, Stay-at-home subjects boxed for a significantly longer period than Control subjects, while on PN34 there was no difference between the two treatment conditions.

\section{DISCUSSION}

Neonatal stress induced by isolation significantly affected play behavior, but these effects varied with the paradigm of isolation. ED subjects demonstrated increased levels of play compared to controls in attacking (both number, total duration and duration per attack), boxing (total duration), and evasions (both number and total duration). ED subjects were not more likely to be supine or submissive. In contrast, MS subjects differed from Controls and ED subjects primarily by engaging in many fewer boxing bouts and less total duration boxing. MS subjects also evaded play interactions more frequently and for a longer duration than Controls.

Differences in the maternal care given upon reunion may account for the differences seen in play behavior. Both the ED and MS procedures altered maternal behavior, but there was a significantly greater effect on the dam in the MS compared to the ED condition. MS dams demonstrated increased nest rebuilding and reorganizing behaviors, relocation of pups, and were more likely to remain active rather than settling into a supine nursing position after $10 \mathrm{~min}$. ED dams also demonstrated elevated maternal behaviors compared to Control dams, suggesting that this paradigm creates a level of stress that is intermediate compared to separation from the entire litter and home nest and the undisturbed condition. In a previous study, using the ED paradigm, dams were observed to lick and groom previously isolated pups a longer time than littermates that had remained in the nest with the dam (Zimmerberg et al., 2003). The MS procedure also alters maternal behavior upon reunion, as dams spent more time LG pups separated briefly ( $15 \mathrm{~min})$ than dams whose pups were not separated (Liu et al., 1997). Boccia and Pedersen (2001) also reported that daily brief ( $15 \mathrm{~min})$ and long $(3 \mathrm{~h})$ separations altered maternal behaviors such as licking, grooming, nursing, and nest-building. Thus, the role of maternal care and variations of the quality of care upon reunion with pups is clearly important in the consideration of separation as a model of early stress.

Our hypothesis stated that differential levels of stress in the dams and pups created by the two paradigms would cause alterations in play differences seen, and the play data are consistent with this hypothesis; the MS model caused the greatest change in maternal behavior of dams upon reunion, while pups demonstrated less alteration in play behaviors than ED pups. Conversely, ED dams displayed less dramatic alterations in maternal behavior, while ED pups displayed more dramatic alterations in play. Attacking (including number of attacks, total duration of attacks, and mean duration per attack) and boxing (also including events, total duration, and mean duration per box), which represent prosocial or aggressive behaviors were significantly increased in ED subjects, but the antisocial or submissive behavior of evasion was also increased. This is not surprising, as every prosocial initiation of play performed by one subject must elicit a response behavior of some sort. Therefore, in a pair where one subject performs more prosocial behaviors, these will be matched by equivalent levels of reciprocating submissive behaviors.

The increased level of boxing demonstrated by ED subjects can be interpreted as elevated aggression. In the case of boxing, both subjects assume aggressive roles and each refuses to "back down" 
into a submissive role. For this reason, an increase in aggressive boxing does not necessarily create a reciprocal increase in submissive behavior. ED subjects therefore demonstrate a disproportionate increase in aggressive compared to submissive behaviors, although both are significantly elevated from Control and MS. Increased boxing behavior can also be interpreted as an impaired readiness or ability to recognize social cues and respond appropriately. Boxing is a much rarer occurrence in play fighting compared to attack-evade or pin-supine interactions. In most play fighting situations, an attack is either met by an evasion or a supine response, which then leads to a continued attack of the evading partner or a pin. Boxing can be viewed as an attack that fails to elicit the more common submissive responses, and the responding partner that reciprocates as failing to interpret the social interaction normally, resulting in a less common, "dysfunctional" interaction.

Early deprivation subjects also displayed increased rearing, suggesting that elevated levels of play could be due to a general increase in activity or arousal. Since ED also causes increased grooming in adults (Zimmerberg et al., 1999), rearing in ED juveniles might be a reflection of changes in affective behaviors which could have an impact on social behavior. General activity is somewhat increased by the ED experience, but play activity is increased proportionately more, reducing the likelihood that general activity changes were responsible for the much greater increases seen in play in ED subjects compared to controls.

Female ED subjects displayed the most boxing (seen in number, total duration and mean duration per box measures) compared to Control females, and even sometimes exceeded levels displayed by ED males. One possible explanation is that upon reunion with the dam, the increased maternal care in response to separation elevated the average amount of licking received per female to levels closer to those of males, causing a masculinizing effect (Birke and Sadler, 1987). As demonstrated previously in this lab, dams can distinguish between separated and non-separated littermates and increased maternal behavior in response to the separated pup (Zimmerberg et al., 2003).

One specific region of the brain that has been implicated in social behavior is the amygdala. Amygdala lesions on PN7 disrupt social behavior in rats later in life while lesions on PN21 have no effect (Wolterink et al., 2001). Rats lesioned on PN7 demonstrated decreased play on PN28, while hippocampal damage on PN7 did not affect play. Amygdala-lesioned rats also demonstrated decreased social exploration and approaching or following behaviors when lesioned on PN7, but not PN21. These results suggest that the amygdala plays a critical role in social and play behavior in juvenile rats, and that there is a critical period of development that occurs early in life. As our results demonstrated increased levels in play, the opposite of that seen after amygdala lesions, it may imply that early separation increases amygdala function or activity.

Previous studies examining the effects of early separation or social isolation have also noted subsequent differences in social behaviors. Arnold and Siviy examined the effects of a 3-h daily separation from PN2-14, using the MS paradigm as described in the current study. They reported that overall levels of playfulness were not affected, but that responsiveness to playful contacts (with evasions or partial rotations) was affected in males. Separated males demonstrated a decrease in evasions as testing progressed over 3 days, while Control males showed no change in evasions. This is consistent with our own results of increased evasions in both MS and ED males compared to controls. It is likely that Siviy and Arnold found fewer differences in play compared to the current study as a function of the separation model used; in the current study, MS differences were indeed more subtle than those of ED. Another possible factor is that in this study, play was measured by the number of contacts to the nape and the responses to those contacts. Therefore, prosocial play behaviors were not distinguished as attacks, pins, or boxes as in the current study, and responses were only characterized as rotations and evasions and supine occurrences were not measured. It is possible that effects seen in the current study on these unrecorded behaviors were masked by their method of play measurement. An additional factor to consider is that the study Arnold and Siviy used pregnant dams that were transported to the lab while pregnant, introducing the possibility of prenatal stress which could also contribute to differences in subsequent pup behavior seen between their own subjects and those in our study, in which the dams were never removed from lab. Veenema and Neumann (2008) reported that MS in males increased aggressive play (attacks, pulling, and biting) and decreased submissive play (supine, evading) compared to controls. There were a number of differences in our procedures, including earlier weaning, group housing after weaning, fewer days of isolation to induce play, and whether the playmate was a littermate. These differences also point to the value in standardizing procedures across laboratories.

Within-litter disturbed controls, here called "stay-at-home" subjects, which remained at home with the dam while ED littermates were separated each day, were also found to have significant differences in play compared to Controls from undisturbed litters. In the measures of attacks, evasions, pins, boxing, supine occurrences and rearing, Stay-at-home subjects differed from Control subjects. The sex-dependent pattern of results suggests a masculinization of play behaviors in females measures and a feminization in males. If these "stay-at-home" subjects received more maternal attention during their time alone with the dam, it further supports the hypothesis that levels of maternal care are essential in the development of later play behaviors. Stay-at-home females may have received more anogenital licking (AGL) than they would have in the larger litter. Typically, dams spend more time licking males than females, and this AGL contributes to masculine development (Birke and Sadler, 1987; Moore and Power, 1992). These results also suggests that Stay-at-home subjects should not be considered equivalent to Control subjects for statistical purposes.

\section{CONCLUSION}

Using a validated animal model of early neglect is critical for determining molecular mechanisms that underlie alterations in juvenile social behavior. The impact of early neglect on later social and stress-response behaviors, as demonstrated in studies such as Chisholm (1998) and Gunnar et al. (2001) on adoptees from Romanian orphanages, reinforces the need for animal experimentation that can determine molecular epigenetic mechanisms of these adverse effects to support new translational treatments of behavioral disorders. Three years following adoption from 
orphanages, Romanian children displayed a higher proportion of insecure social attachments and displayed significantly more indiscriminately friendly behavior toward new adults. Similarly, the current study demonstrated altered social behaviors in juvenile rats who had experienced two different paradigms of early neglect, but these effects varied with the paradigm. ED subjects demonstrated increased levels of play of all types, while MS subjects differed only

\section{REFERENCES}

Arnold, J. L., and Siviy, S. M. (2002). Effects of neonatal handling and maternal separation on rough-andtumble play in the rat. Dev. Psychobiol. 41, 205-212.

Bifulco, A., Brown, G. W., and Adler, Z. (1991). Early sexual abuse and clinical depression in adult life. $\mathrm{Br}$. J. Psychiatry 159, 115-122.

Birke, L., and Sadler, D. (1987). Differences in maternal behavior of rats and the sociosexual development of the offspring. Dev. Psychobiol. 20, 85-99.

Boccia, M. L., and Pedersen, C. A. (2001). Brief versus long maternal separations in infancy: contrasting relationships with adult maternal behavior and lactation levels of aggression and anxiety. Psychoneuroendocrinology 26, 657-672.

Brown, G. R., and Anderson, B. (1991). Psychiatric morbidity in adult inpatients with childhood histories of sexual and physical abuse. Am. J. Psychiatry 148, 55-61.

Caspi, A., Sugden, K., Moffitt, T. E., Taylor, A., Craig, I. W., Harrington, H., McClay, J., Mill, J., Martin, J., Braithwaite, A., and Poulton, R. (2003). Influence of life stress on depression: moderation by a polymorphism in the 5-HTT gene. Science 301, 386-389.

Chisholm, K. (1998). A three year follow-up of attachment and indiscriminate friendliness in children adopted from Romanian orphanages. Child Dev. 69, 1092-1106.

Francis, D. D., Caldji, C., Champagne, F., Plotsky, P. M., and Meaney, M. J. (1999). The role of corticotropinreleasing factor-norepinephrine systems in mediating the effects of early experience on the development of behavioral and endocrine responses to stress. Biol. Psychiatry 46, 1153-1166.

Frisone, D. F., Frye, C. A., and Zimmerberg, B. (2002). Social isolation stress during the third week of life has age-dependent effects on spatial learning in rats. Behav. Brain Res. $128,153-160$.

Gunnar, M., Morrison, S., Chisholm, K., and Schuder, M. (2001). Salivary cortisol levels in children adopted from Romanian orphanages. Dev. Psychopathol. 13, 611-628.

Hofer, M. A. (1987). "Shaping forces within early social relationships," in Perinatal Development: A Psychological Perspective, ed. N. A. Krasnegor (New York: Academic Press), 251-274.

Hofer, M. A. (1996). On the nature and consequences of early loss. Psychosom. Med. 58, 570-581.

Kehoe, P., Mallinson, K., McCormick, C. M., and Frye, C. A. (2000). Central allopregnanolone is increased in rat pups in response to repeated, short episodes of neonatal isolation. Brain Res. Dev. Brain Res. 124 133-136.

Kuhn, C. M., Pauk, J., and Schanberg, S. M. (1990). Endocrine responses to mother-infant separation in developing rats. Dev. Psychobiol. 23, 395-410.

Lehmann, J., and Feldon, J. (2000). Long-term biobehavioral effects of maternal separation in the rat: consistent or confusing? Rev. Neurosci. 11, 383-408.

Lehmann, J., Pryce, C. R., Brettschen, D., and Feldon, J. (1999). The maternal separation paradigm and adult emotionality and cognition in male and female Wistar rats. Pharmacol. Biochem. Behav. 64, 705-715.

Levy, F., Melo, A. I., Galef, B. G., Madden, M., and Fleming, A. S. (2003). Complete maternal deprivation affects social, but not spatial, learning in adult rats. Dev. Psychobiol. 43, 177-191.

Liu, D., Diorio, J., Day, J. C., Francis, D. D., and Meaney, M. J. (2000). Maternal care, hippocampal synaptogenesis and cognitive development in rats. Nat. Neurosci. 3 , 799-806.

Liu, D., Diorio, J., Tannenbaum, B. Caldji, C., Francis, D., Freedman, A., Sharma, S., Pearson, D., Plotsky, P. M., and Meaney, M. J. (1997). Maternal care, hippocampal glucocorticoid receptors, and hypothalamic-pituitary-adrenal responses to stress. Science 277, 1659-1662.

Menard, J. L., and Hakvoort, R. M. (2007). Variations in materal

primarily by engaging in less boxing and more evading. Using the ED model might maximize the behavioral outcome by stressing the stressed individual while minimizing, although certainly not eliminating, effects that are maternally mediated. Further research on early separation and subsequent effects on social behavior would benefit from careful attention to the model of early stress and the role of maternal care.

care alter offspring levels of behavioral defensiveness in adulthood. Evidence for a threshold model. Behav. Brain Res. 176, 302-313.

Moore, C., and Power, K. (1992). Variation in maternal care and individual differences in play, exploration, and grooming of juvenile Norway rat offspring. Dev. Psychobiol. 25, 165-182.

O'Connor, T. G., and Rutter, M. (2000). Attachment disorder behavior following early severe deprivation: extension and longitudinal followup. J. Am. Acad. Child Adolesc. Psychiatry 39, 703-712.

Parent, C. I., and Meaney, M. J. (2008). The influence of natural variations in maternal care on play fighting in the rat. Dev. Psychobiol. 50, 767-776.

Pellis, S. M., Field, E. F., Smith, L. K., and Pellis, V. C. (1997). Multiple differences in the play fighting of male and female rats. Implications for the causes and functions of play. Neurosci. Biobehav. Rev. 21 , 105-120.

Pellis, S. M., and Pellis, V. C. (1990). Differential rates of attack, defense and counterattack during the developmental decrease in play fighting by male and female rats. Dev. Psychobiol. 23 215-231.

Pellis, S. M., and Pellis, V. C. (1998). Play fighting of rats in comparative perspective: a schema for neurobehavioral analyses. Neurosci. Biobehav. Rev. 23, 87-101.

Pryce, C. R., Bettschen, D., NanzBahr, N. I., and Feldon, J. (2003). Comparison of the effects of early handling and early deprivation on conditioned stimulus, context, and spatial learning and memory in adult rats. Behav. Neurosci. 117, 883-893.

Sanchez, M. M., Ladd, C. O., and Plotsky, P. M. (2001). Early adverse experience as a developmental risk factor for later psychopathology: evidence from rodent and primate models. Dev. Psychobiol. 13, 419-449.

Sandstrom, N. J., and Hart, S. R. (2005). Isolation stress during the third postnatal week alters radial arm maze performance and corticosterone levels in adulthood. Behav. Brain Res. 156, 289-296.

Stanton, M. E., Gutierrez, Y. R., and Levine, S. (1988). Maternal deprivation potentiates pituitary-adrenal stress responses in infant rats. Behav. Neurosci. 102, 692-700.

Stanton, M. E., and Levine, S. K (1990). Inhibition of infant glucocorticoid stress response: specific role of maternal cues. Dev. Psychobiol. 23, 411-426.

Teicher, M. H., Andersen, S. L., Polcari, A., Anderson, C. M., and Navalta, C. P. (2002). Developmental neurobiology of childhood stress and trauma. Psychiatr. Clin. North Am. 25, 397-426.

Thor, D. H., and Holloway, W. R. (1984). Social play in juvenile rats: a decade of methodological and experimental research. Neurosci. Biobehav. Rev. 8 , 455-464.

Veenema, A. H., and Neumann, I. D. (2008). Maternal separation enhances offensive play-fighting, basal corticosterone and hypothalamic vasopressin mRNA expression in juvenile male rats. Psychoneuroendocrinology 34 463-467.

Wolterink, G., Daene, L., Dubbledam, S., Gerrits, M., Rijn, R., Kruse, C., Van Der Heijden, J., and Van Ree, J. (2001). Early amygdala damage in the rat as a model for neurodevelopmental psychopathological disorders. Eur. Neuropsychopharmacol. 11, 51-59.

Zeanah, C. H. (2000). Disturbances of attachment in young children adopted from institutions. J. Dev. Behav. Pediatr. 21, 230-236.

Zeanah, C. H., Smyke, A. T., and Dumitrescu, A. (2002). Attachmen disturbances in young children. II. Indiscriminate behavior and institutional care. J. Am. Acad. Child Adolesc. Psychiatry 41, 983-989.

Zimmerberg, B., Rackow, S. H., and George-Friedman, K. P. (1999). Sex-dependent behavioral effects of the neurosteroid allopregnanolone (3alpha, 5alpha-THP) in neonatal and adult rats after postnatal 
stress. Pharmacol. Biochem. Behav. 64, 717-724.

Zimmerberg, B., Rosenthal, A. J., and Stark, A. C. (2003). Neonatal social isolation alters both maternal and pup behaviors in rats. Dev. Psychobiol. 42, 52-63.

Zimmerberg, B., and Shartrand, A. M. (1992). Temperature-dependent effects of maternal separation on growth, activity, and amphetamine sensitivity in the rat. Dev. Psychobiol. 25, 213-226.

Conflict of Interest Statement: The authors declare that the research was conducted in the absence of any commercial or financial relationships that could be construed as a potential conflict of interest.
Received: 24 March 2011; accepted: 14 June 2011; published online: 28 June 2011.

Citation: Zimmerberg B and Sageser KA (2011) Comparison of two rodent models of maternal separation on juvenile social behavior. Front. Psychiatry 2:39. doi: 10.3389/fpsyt.2011.00039

This article was submitted to Frontiers in Child and Neurodevelopmental
Psychiatry, a specialty of Frontiers in Psychiatry.

Copyright (c) 2011 Zimmerberg and Sageser. This is an open-access article subject to a non-exclusive license between the authors and Frontiers Media SA, which permits use, distribution and reproduction in other forums, provided the original authors and source are credited and other Frontiers conditions are complied with. 